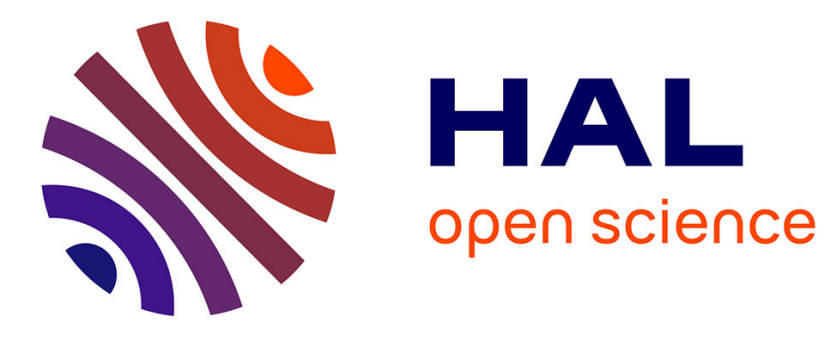

\title{
Multispectral constancy based on spectral adaptation transform
}

\author{
Haris Ahmad Khan, Jean-Baptiste Thomas, Jon Yngve Hardeberg
}

\section{To cite this version:}

Haris Ahmad Khan, Jean-Baptiste Thomas, Jon Yngve Hardeberg. Multispectral constancy based on spectral adaptation transform. Scandinavian Conference on Image Analysis, Jun 2017, Troms $\varnothing$, Norway. pp.459-470, 10.1007/978-3-319-59129-2_39 . hal-01672532

\section{HAL Id: hal-01672532 \\ https://hal.science/hal-01672532}

Submitted on 26 Dec 2017

HAL is a multi-disciplinary open access archive for the deposit and dissemination of scientific research documents, whether they are published or not. The documents may come from teaching and research institutions in France or abroad, or from public or private research centers.
L'archive ouverte pluridisciplinaire HAL, est destinée au dépôt et à la diffusion de documents scientifiques de niveau recherche, publiés ou non, émanant des établissements d'enseignement et de recherche français ou étrangers, des laboratoires publics ou privés. 


\title{
Multispectral constancy based on spectral adaptation transform
}

\author{
Haris Ahmad Khan ${ }^{1,2}$, Jean Baptiste Thomas ${ }^{1,2}$, and Jon Yngve Hardeberg ${ }^{1}$ \\ ${ }^{1}$ The Norwegian Colour and Visual Computing Laboratory, \\ NTNU - Norwegian University of Science and Technology, Gjøvik, Norway \\ ${ }^{2}$ Le2i, FRE CNRS 2005, Univ. Bourgogne Franche-Comté, Dijon, France \\ \{haris.a.khan, jean.b.thomas, jon.hardeberg\}@ntnu.no
}

\begin{abstract}
The spectral reflectance of an object surface provides the valuable information about its characteristics. Reflectance reconstruction is based on certain assumptions. One of these assumptions is that the same illumination is used for system calibration and the image acquisition. We propose multispectral constancy through spectral adaptation transform, which transforms the sensor data acquired under an unknown illumination to a generic illuminant-independent space, similar to the computational color constancy. Spectral reflectance is then estimated by using a generic linear calibration. Results of reflectance reconstruction using the proposed concept show that this concept is efficient, but highly sensitive to the accuracy of illuminant estimation.
\end{abstract}

Keywords: Reflectance reconstruction, multispectral constancy, illuminant estimation, spectral adaptation transform

\section{Introduction}

Spectral imaging (SI) refers to the acquisition of image data at specific intervals in the electromagnetic spectrum by the use of spectral filters. Color images contain three channels while a spectral image consists of more than three channels. SI was originally developed for remote sensing applications [1. With advancement in sensor technology, use of SI for indoor scene acquisition under controlled conditions has also increased. The advantage of SI is the ability to acquire more spectral information of a scene, which can be used for spectral reflectance reconstruction [2] of the object's surfaces. One of the limitations associated with SI is the need of system calibration for the imaging environment when performing the spectral reconstruction [3. A system being calibrated for one type of conditions needs to be re-calibrated if it has to be used in another imaging environment. One of the major limitation is the need for having the same illuminant for spectral reconstruction system's calibration and the image acquisition. This limitation is a major challenge and hurdle for the use of SI in outdoor environments.

In this paper, we introduce the concept of multispectral constancy for SI. We define multispectral constancy similarly to the color constancy 4 but in higher spectral dimension. By multispectral constancy, we refer to representation 
of imaged surfaces without the effect of scene illuminant. In other words, the surfaces appear as if they are taken under a canonical illuminant. To achieve multispectral constancy, we introduce a spectral adaptation transform (SAT). The closest concept to SAT in the literature is defined as spectral adaptation, being applied on spectra by Fairchild [5, while we generalize it to the sensor measurements. By attaining multispectral constancy, SI can be used for any illuminant without the requirement of re-calibration when imaging environment is changed.

This paper is organised as follows. Section 2 formalises the problem of spectral acquisition, including the calibration and spectral reconstruction as a linear problem. We propose to add a term to the usual calibration, which discard the illumination changes. Section 3 defines our simulation and experimental protocol. Results are analyzed in Section 4 before we conclude.

\section{Spectral Adaptation Transform}

In the context of a simplified imaging model, a pixel being captured in the imaging system at a location $(i, j)$, is the combination of spectral reflectance of the surface $R(i, j)(\lambda)$, spectral power distribution of illuminant $E(\lambda)$ and spectral sensitivity of the $n^{t h}$ spectral filter $m_{n}(\lambda)$. This formation for the visible wavelength spectrum $\omega$ is defined in Eq. 1 .

$$
F_{n}(i, j)=\int_{\omega} R(i, j)(\lambda) E(\lambda) m_{n}(\lambda) d \lambda
$$

In practice, we can formulate a discrete version of Eq. 1 as $\mathbf{F}=\mathbf{R E M}$, where $\mathbf{M}=\left\{m_{1}(\lambda), m_{2}(\lambda), \ldots, m_{N}(\lambda)\right\}$ and $N$ is the total number of filters.

Here we consider two cases of image acquisition. One is with a canonical illumination $E_{c}$ and the other case is when an unknown illuminant $E_{i l l}$ is used. We present both cases in parallel in Equation 2.

$$
\mathbf{F}_{i l l}=\mathbf{R E}_{i l l} \mathbf{M} \quad ; \quad \mathbf{F}_{c}=\mathbf{R E}_{c} \mathbf{M}
$$

To perform the spectral reflectance estimation $\hat{\mathbf{R}}$ from the imaged data in both of the above mentioned cases, we can apply a generalized inverse, noticed ${ }^{+}$, as in Equation 3

$$
\hat{\mathbf{R}}=\mathbf{F}_{i l l} \mathbf{M}^{+} \mathbf{E}_{i l l}^{+} \quad ; \quad \hat{\mathbf{R}}=\mathbf{F}_{c} \mathbf{M}^{+} \mathbf{E}_{c}^{+}
$$

For the reconstruction of spectral reflectance, knowledge of illumination is required. There are many methods to estimate the sensor sensitivity $\mathbf{M}$ for a given camera [6] [7. However, it is not an easy task to measure the scene illuminant every time along with the image acquisition.

The spectral reconstruction is performed by training a calibration matrix $\mathbf{W}=\mathbf{M}^{+} \mathbf{E}^{+}$8]. This calibration is specific for a given illumination $E_{c}$. By using the calibration matrix $\mathbf{W}$, the equations for spectral reflectance reconstruction become $\hat{\mathbf{R}}=\mathbf{F}_{i l l} . \mathbf{W}_{i l l}$ and $\hat{\mathbf{R}}=\mathbf{F}_{c} \cdot \mathbf{W}_{c}$ for both cases, respectively. We propose 
to transform the acquired image $\mathbf{F}_{i l l}$ into a canonical representation $\mathbf{F}_{c}$. In this way, $\mathbf{W}_{c}$ can be used for the spectral reconstruction from a multispectral image, being taken under any illumination. We call this concept as the multispectral constancy. It is achieved through SAT. This transformation is in the form of a diagonal matrix $\mathbf{A}_{S A T}$ and is applied as,

$$
\mathbf{F}_{c}=\mathbf{A}_{S A T} \mathbf{F}_{i l l}
$$

In Eq. 4. SAT being applied to the acquired spectral data enables the estimation of reflectance spectra from an image being taken under any illuminant and can be used as,

$$
\hat{\mathbf{R}}=\mathbf{W}_{c} \mathbf{A}_{S A T} \mathbf{F}_{i l l}
$$

With the use of SAT as in Eq. 5, the requirement of having same illuminant for spectral reconstruction system's calibration and the image acquisition, is no longer required since the acquired spectral image is transformed before the estimation of spectra. Obtaining $\mathbf{A}_{S A T}$ and $\mathbf{W}$ are explained in Sections 3.3 and 3.4 , respectively.

\section{Simulation}

\subsection{Simulation pipeline}

Figure 1 shows the pipeline of experimental framework for the spectral reconstruction, based on multispectral constancy. This pipeline consists of sensor simulation, acquisition of spectral data, SAT, spectral reconstruction and the evaluation of results. These blocks are explained in the following sections.

\subsection{Sensor}

To implement and validate the proposed idea of multispectral constancy, we use reflectance data in the wavelength range $R=\{400 \mathrm{~nm}, 410 \mathrm{~nm} \ldots, 720 \mathrm{~nm}\}$, from the GretagMacbeth ColorChecker [9]. We apply equi-Gaussian filters [10] for simulation of the spectral filters and use 8 filters in the experiments. By increasing the number of filters, more noise is introduced in the image which effects the spectral reconstruction. This effect was observed by Wang et al. 8]. Radiance data is created by using the illuminants E and D65 and then the simulated sensors are used to acquire the multispectral data.

\subsection{Multispectral constancy through SAT}

In order to achieve multispectral constancy, SAT is applied to the spectral image after the estimation of illuminant in the sensor domain as in Eq. 5 . For estimation of the illuminant in spectral images of natural scenes, we propose using the MaxSpectral Algorithm and the Spectral Gray-Edge Algorithm. These algorithms are 


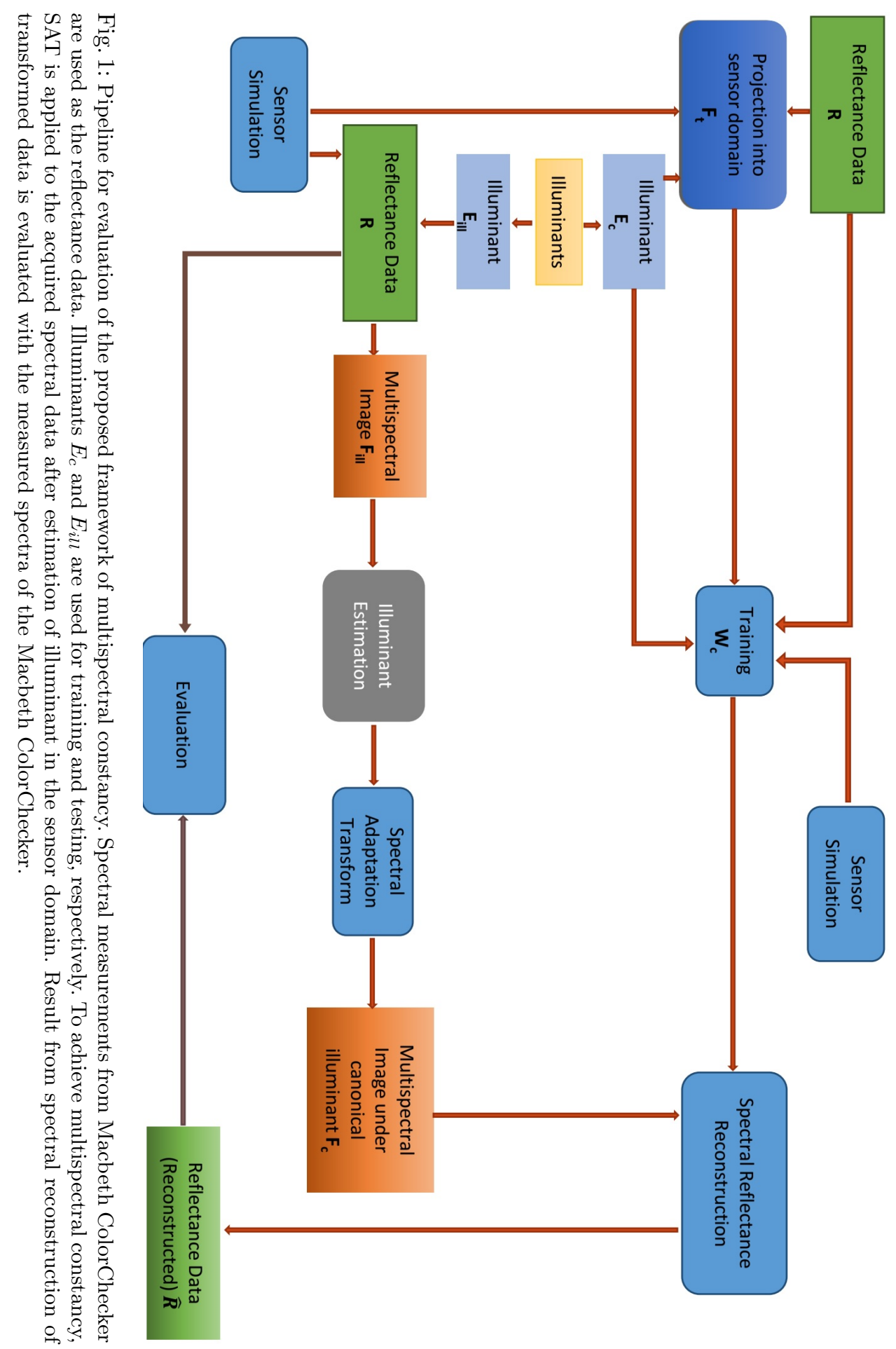


extensions of Max-RGB Algorithm [11] and the Gray-Edge Algorithm [12 13. The extension of these algorithms from color to spectral is discussed in detail in [14]. Once the illuminant is estimated, then we propose to apply the SAT in form of a diagonal correction to the acquired data, so that it appears as if being taken under a canonical illuminant. Such a diagonal transform for color images was initially proposed by Von Kries [15]. We extend this transform into the spectral domain. For $N$ number of channels, SAT is defined as in Eq. 6 .

$$
\left(\begin{array}{c}
F_{1}^{c} \\
F_{2}^{c} \\
\vdots \\
F_{N}^{c}
\end{array}\right)=\left(\begin{array}{cccc}
K_{1} & 0 & \ldots & 0 \\
0 & K_{2} & \ldots & 0 \\
\vdots & \vdots & \ddots & \vdots \\
0 & 0 & \ldots & K_{N}
\end{array}\right) \cdot\left(\begin{array}{c}
F_{1}^{u} \\
F_{2}^{u} \\
\vdots \\
F_{N}^{u}
\end{array}\right)
$$

Here, $\mathbf{F}_{n}^{u}$ is the pixel of $n^{t h}$ channel, taken under an unknown illuminant while $\mathbf{F}_{n}^{c}$ is the transformed images so that it appears to be taken under a canonical illuminant. $K_{n}$ is the correction parameter for the channel $n$ which is obtained from the illuminant estimation 14 .

\subsection{Spectral reflectance reconstruction}

As explained in Section 2, a training matrix is required for the spectral reconstruction from camera data. This matrix $\mathbf{W}$ is called the calibration matrix. It is obtained by using measured reflectance spectra $\mathbf{R}_{t}$ and the acquisition of radiance images $\mathbf{F}_{t}$, using the same camera. We use reflectance data from the GretagMacbeth ColorChecker [9] for $\mathbf{R}_{t}$ and the acquisition of $\mathbf{F}_{t}$.

There are many methods being proposed for spectral reconstruction. We use linear methods to keep the proposed system simple and robust. Those linear methods include the linear least square regression, principal component analysis [16] and Wiener estimation [17. We checked the performance of these three methods and got similar results. For this paper, we decide to use Wiener estimation because it is robust to noise and fulfills the criteria of being linear. It is defined as in Eq. 7

$$
\mathbf{W}=\mathbf{F}_{t} \mathbf{F}_{t}^{T}\left(\mathbf{R}_{t} \mathbf{E}\right)^{t}\left(\left(\mathbf{R}_{t} \mathbf{E}\right) \mathbf{F}_{t} \mathbf{F}_{t}^{T}\left(\mathbf{R}_{t} \mathbf{E}\right)^{T}+A\right)^{+}
$$

Here, $A$ is the term used for additive noise.

One of the major shortcoming of the linear methods for spectral reconstruction is the assumption that the same illuminant is used for system's calibration and acquisition of data. We are interested in the development of a spectral reconstruction system which do not require the same illuminant for system's calibration and the data acquisition. This can be achieved through the illuminant estimation in spectral images and then applying SAT to the acquired data as in Eq. 5. The matrix $\mathbf{W}$ is obtained by using the training reflectance spectra and radiance with the canonical illuminant $E_{c}$ (we use illuminant E). This matrix is used for the spectral reconstruction from the spectral data being taken under any lighting conditions. 
To check the validity of our proposed idea, we perform experiments on the measured reflectance data of Macbeth ColorChecker for spectral reflectance reconstruction. Those results are provided in Section 4.

\subsection{Evaluation}

For evaluation of the performance of spectral reconstruction $\hat{\mathbf{R}}$, we compare the results with measured spectra $\mathbf{R}$ through root mean square error (RMSE) and Goodness of fit coefficient (GFC) as in Eq. 8 and 9 respectively.

$$
\begin{gathered}
\mathrm{RMSE}=\sqrt{\frac{1}{N} \sum|\mathbf{R}-\hat{\mathbf{R}}|} \\
\mathrm{GFC}=\frac{\left\|\sum \mathbf{R} \cdot \hat{\mathbf{R}}\right\|}{\left\|\sum \mathbf{R}^{2}\right\|^{\frac{1}{2}} \cdot\left\|\sum \hat{\mathbf{R}}^{2}\right\|^{\frac{1}{2}}}
\end{gathered}
$$

Besides that, we also provide spectral reconstruction results for the reflectance patches of the Macbeth ColorChecker in the form of graphs so that the overall performance of spectral reconstruction can be visually analyzed.

\section{Results}

In this section we evaluate the validity of our proposed idea of multispectral constancy through SAT. We also investigate the influence of illuminant estimation on the results of spectral reconstruction. We use three different noisy estimates of illuminant for testing the proposed framework to assess the influence of erroneous illuminant estimation.

For training of matrix W, 24 patches of Macbeth ColorChecker are used within the visible wavelength range (400-720 nm). For spectral reconstruction, we use different scenarios which are given in Table 1 .

For testing the influence of error in the illuminant estimation, we use three different noisy estimates. Angular error $(\Delta A)$ is calculated in term of radians between the original illuminant $\mathbf{e}$ and the estimated illuminant $\hat{\mathbf{e}}$ as

$$
\Delta A=\arccos \frac{\mathbf{e} . \hat{\mathbf{e}}}{\|\mathbf{e}\| \cdot\|\hat{\mathbf{e}}\|}
$$

We use three different noisy estimates of illuminant with $\Delta A$ of $0.0210,0.1647$ and 0.3658 radians. For checking the effect of illuminant estimation, we apply these noisy illuminants in the sensor domain to the acquired spectral image of Macbeth ColorChecker according to Eq. 6. First we evaluate spectral reconstruction and then analyze the performance of SAT. Figures 2,3 and 4 show results obtained from the 24 reflectance patches of the Macbeth Colorchecker in the visible spectrum. These results are discussed in the following sections. 
Table 1: Description of the experiments being performed. Adequate calibration of system is performed by using the same illuminant. In no correction case, we use different illuminants and do not apply any correction. In ideal correction, SAT is applied by assuming efficient estimation of the illuminant while in naive correction, SAT is applied using the illuminant E, while D65 is used for image acquisition. In next three experiments, we apply SAT using the three different noisy estimate of illuminants.

\begin{tabular}{|c|c|c|c|c|}
\hline Experiment & $\begin{array}{c}\text { Illuminant for } \\
\text { training }\end{array}$ & $\begin{array}{c}\text { Illuminant } \\
\text { for SI }\end{array}$ & $\begin{array}{c}\text { SAT } \\
\text { Applied }\end{array}$ & Illuminant for SAT \\
\hline $\begin{array}{c}\text { Adequate } \\
\text { Calibration }\end{array}$ & $\mathrm{D} 65$ & $\mathrm{D} 65$ & No & - \\
\hline No Correction & $\mathrm{E}$ & $\mathrm{D} 65$ & No & - \\
\hline Ideal Correction & $\mathrm{E}$ & $\mathrm{D} 65$ & Yes & $\mathrm{D} 65$ \\
\hline Naive Correction & $\mathrm{E}$ & $\mathrm{D} 65$ & Yes & $\mathrm{E}$ \\
\hline Noisy estimate 1 & $\mathrm{E}$ & $\mathrm{D} 65$ & Yes & $\begin{array}{l}\text { Estimated illuminant } \\
\text { with } \Delta A=0.0210 \mathrm{rad}\end{array}$ \\
\hline Noisy estimate 2 & $\mathrm{E}$ & $\mathrm{D} 65$ & Yes & $\begin{array}{l}\text { Estimated illuminant } \\
\text { with } \Delta A=0.1647 \mathrm{rad}\end{array}$ \\
\hline Noisy estimate 3 & $\mathrm{E}$ & $\mathrm{D} 65$ & Yes & $\begin{array}{l}\text { Estimated illuminant } \\
\text { with } \Delta A=0.3658 \mathrm{rad}\end{array}$ \\
\hline
\end{tabular}

\subsection{Reflectance estimation}

With the use of linear method for spectral reconstruction (Wiener estimation [17), we evaluate the performance of the algorithm using the adequate calibration of system. We check the performance with both illuminants E and D65. They provide similar results. We show results of adequate calibration with illuminant D65 in Fig. 2, 3 and 4. This is the best reconstruction that can be obtained with this given number of sensors and sensor configuration. We investigate the performance of Wiener estimation when different illuminants are used for training and testing and no SAT is applied. We also perform SAT with illuminant $\mathrm{E}$ in sensor domain and the camera data being acquired with illuminant D65. We call this as naive correction. With adequate calibration, the best spectral reconstruction results we could obtain with Wiener estimator, provided an average RMSE of 0.001 and average GFC of 0.9993 over the 24 patches of Macbeth ColorChecker. With no correction being applied and using different illuminants for training and acquisition, average RMSE and GFC were 0.0118 and 0.9898 respectively. To validate the idea of multispectral constancy through SAT, the error in spectral reconstruction must be low as compared to the error in case of applying no correction. We evaluate the performance of SAT in Section 4.2.

\subsection{SAT performance}

Figures 2, 33 and 4, show the spectral reconstruction results from seven different experiments as given in Table 2. Although exact spectral reconstruction is not possible with reduced number of bands, Wiener estimation method is still able 
Table 2: RMSE and GFC from spectral reconstruction of 24 patches of the Macbeth ColorChecker.

\begin{tabular}{|c|c|c|}
\hline Experiment & RMSE & GFC \\
\hline Adequate Calibration & 0.0011 & 0.9993 \\
\hline No Correction & 0.0118 & 0.9898 \\
\hline Ideal Correction & 0.0081 & 0.9973 \\
\hline Naive Correction & 0.0091 & 0.9939 \\
\hline Noisy estimate 1 & 0.0106 & 0.9955 \\
\hline Noisy estimate 2 & 0.0128 & 0.9853 \\
\hline Noisy estimate 3 & 0.0240 & 0.9437 \\
\hline
\end{tabular}

to make a close match when the same illuminant $\mathrm{E}$ is used for both training and acquisition. We test SAT by using illuminant $\mathrm{E}$ for training the matrix $\mathbf{W}$ and the acquired spectral data from the Macbeth ColorChecker after creation of radiance data with D65 illuminant. SAT is applied to the acquired spectral image and then spectral reconstruction is performed. It is obvious from the spectral reconstruction results that the overall accuracy of our proposed framework is dependant on the accuracy of illuminant estimation. With efficient illuminant estimation, SAT is almost the same efficient as in the case of adequate calibration. However, it is interesting to note that although there is overlapping between the spectral reflectance reconstruction curves in the case of adequate calibration and ideal correction as seen in Figures 2, 3 and 4 , the difference in RMSE and GFC is not as close as expected (see Table 2). This leads to opening the discussion about efficiency of SAT and the fact that should SAT be optimised as well in order to get more efficient results. Another problem to be investigated is the required efficiency of both SAT and the illuminant estimation for applications like object detection and classification on the basis of their spectral properties. However, the closeness in result proves that if efficient illuminant estimation is performed and SAT is applied, we can attain close results as compared with adequate calibration. Our proposed idea is valid and there is no need to acquire SPD of the scene illuminant explicitly. The only factor which remains important in our proposed idea of multispectral constancy is the efficient estimation of illuminant in the spectral image.

The dependence of SAT on accuracy of illuminant estimation is clear when spectral reconstruction is performed after applying SAT using the noisy illuminants. In each case, there is error in the illuminant estimation and this error is escalated further during the spectral reconstruction. In these cases, the error in spectral reconstruction is worst from the instance when no correction is applied. It is interesting to note that even the naive correction is able to perform well, which makes the role of illuminant estimation as a significant factor for our proposed idea of multispectral constancy through SAT. SAT itself also needs to be investigated so that an efficient framework for the transformation of acquired 
spectral image into the illuminant free representation (multispectral constancy) can be achieved.

\section{Conclusion}

This work formalizes the concept of multispectral constancy, which permits spectral image acquisition, independent of the illumination. Multispectral constancy is achieved via a spectral adaptation transform, which changes data representation from the actual sensor domain, towards a canonical one, where calibration applies.

Simulation results show that a diagonal SAT permits to achieve similar reflectance reconstruction than when the samples are acquired under the illumination being used for calibration. However, when the spectral adaptation transform is evaluated based on an estimate of illumination, error in illuminant estimates makes the performance to drop down greatly.

It is still to be investigated that what accuracy is required for the illuminant estimation to make this concept beneficial. It is also important to recall that these results are compiled based on simulation without noise during the sensor data acquisition. Further work shall investigate these directions and define the limits of using this approach.

\section{References}

1. R. A. Schowengerdt, Remote Sensing, Third Edition: Models and Methods for Image Processing. Orlando, FL, USA: Academic Press, Inc., 2006.

2. J. Y. Hardeberg, Acquisition and Reproduction of Color Images: Colorimetric and Multispectral Approaches. Universal Publishers, 2001.

3. N. Shimano and M. Hironaga, "Recovery of spectral reflectances of imaged objects by the use of features of spectral reflectances," J. Opt. Soc. Am. A, vol. 27, pp. 251258, Feb 2010.

4. D. H. Foster, "Color constancy," Vision Research, vol. 51, no. 7, pp. 674-700, 2011.

5. M. D. Fairchild, "Spectral adaptation," Color Research \& Application, vol. 32, no. 2, pp. 100-112, 2007.

6. J. Jiang, D. Liu, J. Gu, and S. Süsstrunk, "What is the space of spectral sensitivity functions for digital color cameras?," in IEEE Workshop on Applications of Computer Vision (WACV), pp. 168-179, Jan 2013.

7. G. D. Finlayson, S. Hordley, and P. M. Hubel, "Recovering device sensitivities with quadratic programming," in Color and Imaging Conference, no. 1, pp. 90-95, 1998.

8. X. Wang, J.-B. Thomas, J. Y. Hardeberg, and P. Gouton, "Multispectral imaging: narrow or wide band filters?," Journal of the International Colour Association, vol. 12 , pp. 44-51, July 2014.

9. C. S. McCamy, H. Marcus, and J. G. Davidson, "A color-rendition chart," Journal of Applied Photographic Engineering, vol. 2, no. 3, pp. 95-99, 1976.

10. J.-B. Thomas, "Illuminant estimation from uncalibrated multispectral images," in Colour and Visual Computing Symposium (CVCS), pp. 1-6, Aug 2015.

11. E. H. Land and J. J. McCann, "Lightness and retinex theory," J. Opt. Soc. Am., vol. 61, pp. 1-11, Jan 1971. 


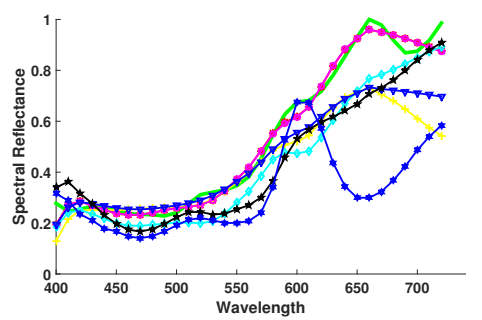

(a) Reflectance patch 1

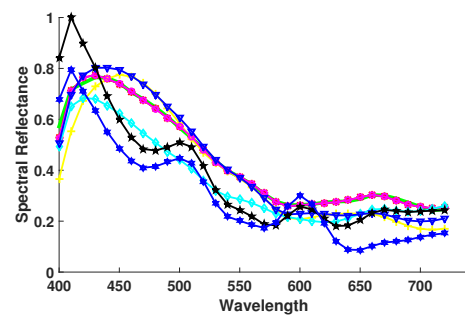

(c) Reflectance patch 3

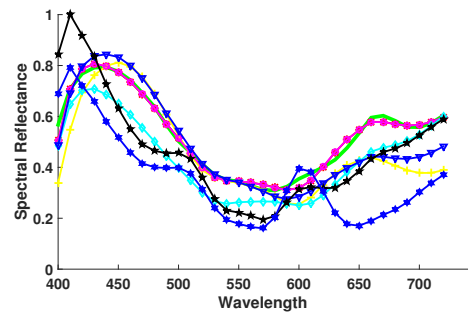

(e) Reflectance patch 5

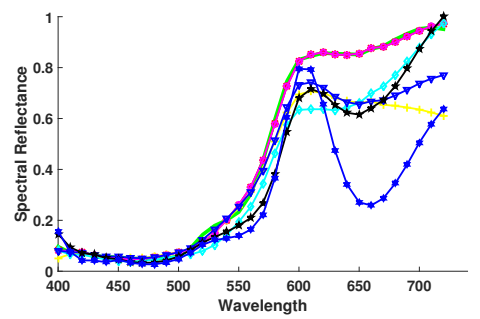

(g) Reflectance patch 7

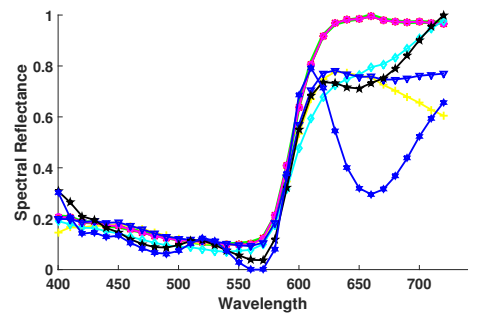

(i) Reflectance patch 9

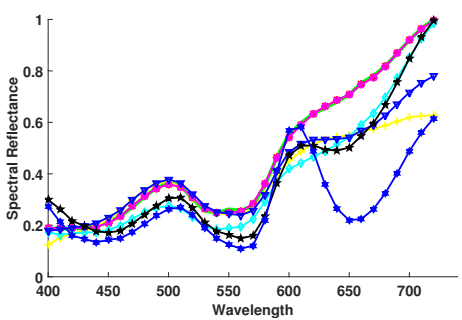

(b) Reflectance patch 2

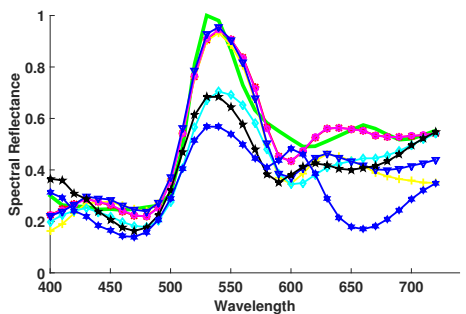

(d) Reflectance patch 4

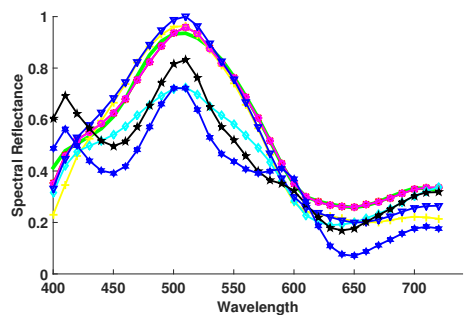

(f) Reflectance patch 6

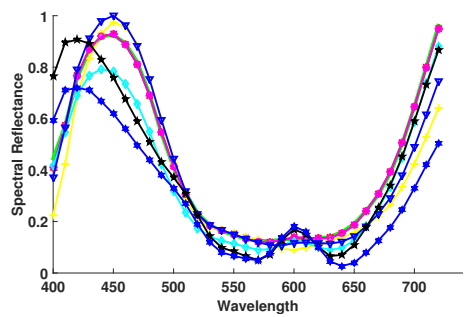

(h) Reflectance patch 8

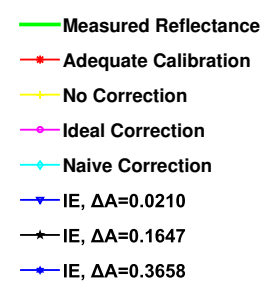

(j) Legend

Fig. 2: First 9 reflectance patches from Macbeth ColorChecker. In each figure, there are curves of measured reflectance, spectral reconstruction from adequate calibration, no correction, ideal correction, naive correction, reflectance reconstruction results after applying SAT from estimated illuminants having $\Delta A$ of $0.0210,0.1647$ and 0.3658 , respectively. Fig. (j) shows the legend. 


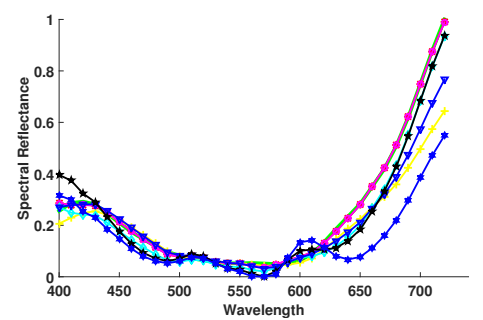

(a) Reflectance patch 10

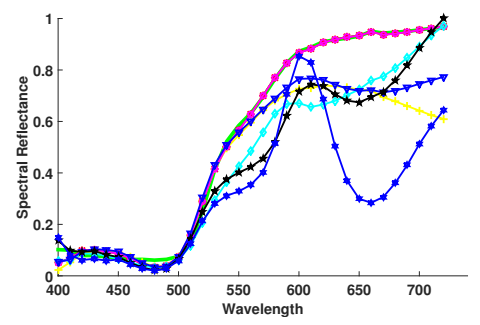

(c) Reflectance patch 12

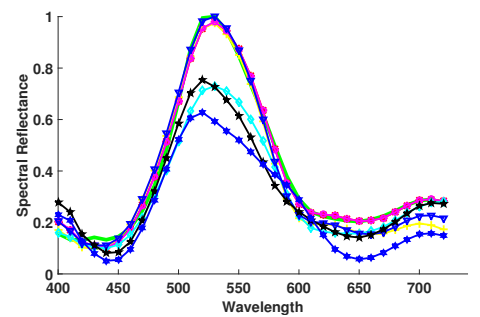

(e) Reflectance patch 14

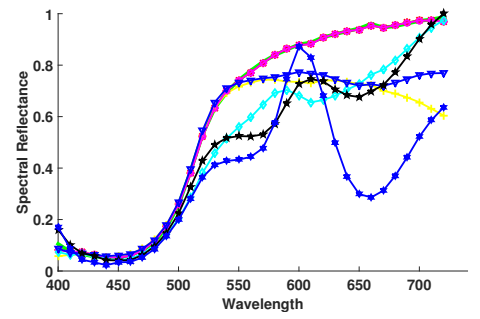

(g) Reflectance patch 16

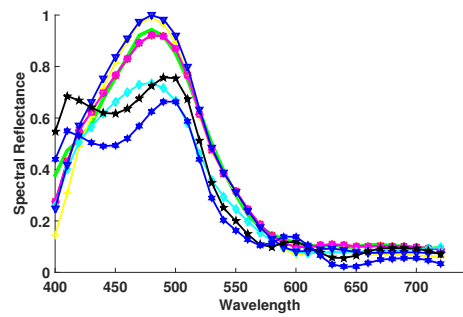

(i) Reflectance patch 18

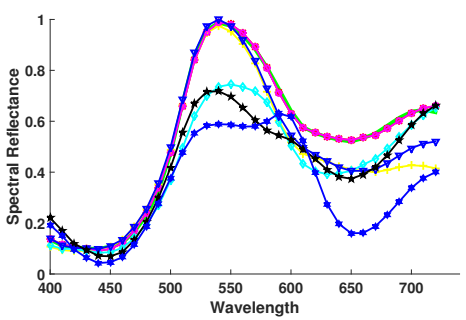

(b) Reflectance patch 11

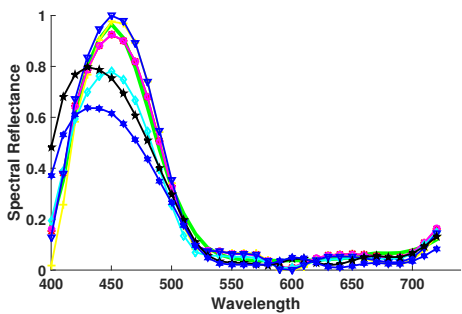

(d) Reflectance patch 13

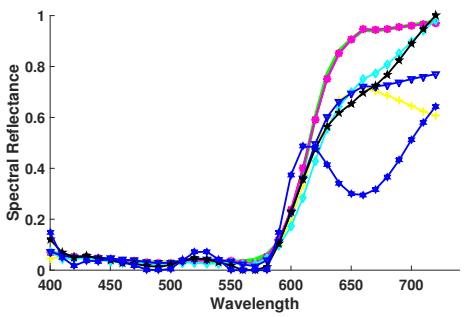

(f) Reflectance patch 15

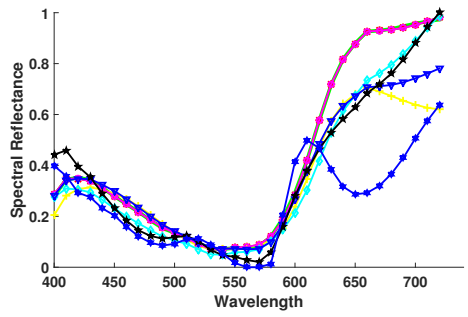

(h) Reflectance patch 17

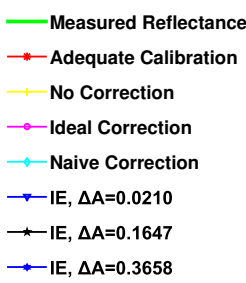

(j) Legend

Fig. 3: Reflectance patches 10-18 from the Macbeth ColorChecker. In each figure, there are curves of measured reflectance, spectral reconstruction from adequate calibration, no correction, ideal correction, naive correction, reflectance reconstruction results after applying SAT from estimated illuminants having $\Delta A$ of $0.0210,0.1647$ and 0.3658 , respectively. Fig. (j) shows the legend. 


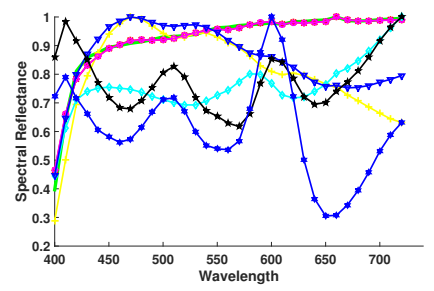

(a) Reflectance patch 19

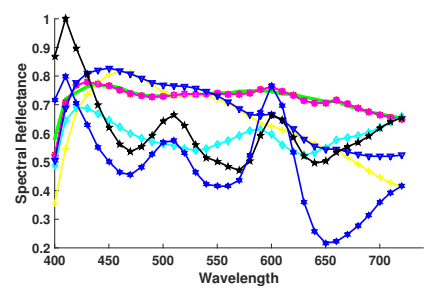

(c) Reflectance patch 21

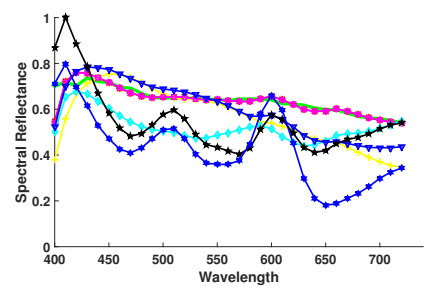

(e) Reflectance patch 23

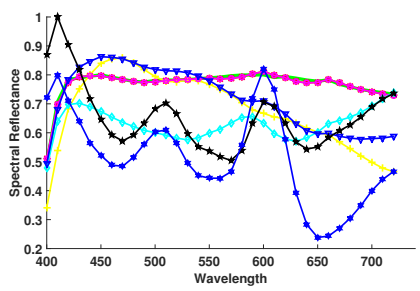

(b) Reflectance patch 20

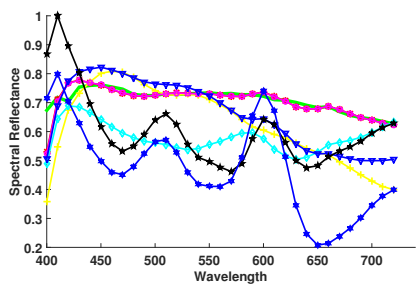

(d) Reflectance patch 22

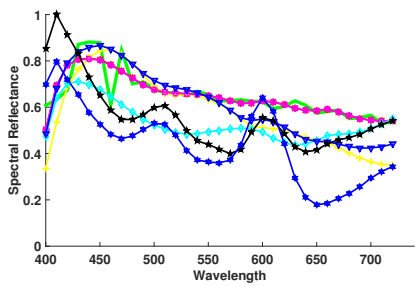

(f) Reflectance patch 24

Fig. 4: Reflectance patches 19-24 from the Macbeth ColorChecker.

12. J. van de Weijer and T. Gevers, "Color constancy based on the Grey-edge hypothesis," in IEEE International Conference on Image Processing, vol. 2, pp. II-722-5, Sept 2005.

13. J. van de Weijer, T. Gevers, and A. Gijsenij, "Edge-based color constancy," IEEE Transactions on Image Processing, vol. 16, pp. 2207-2214, Sept 2007.

14. H. A. Khan, J.-B. Thomas, J. Y. Hardeberg, and O. Laligant, "Illuminant estimation in multispectral imaging," Submitted in Journal for review.

15. J. von Kries, "Influence of adaptation on the effects produced by luminous stimuli," Sources of color vision, MIT Press, Cambridge, pp. 109-119, 1970.

16. F. H. Imai and R. S. Berns, "Spectral estimation using trichromatic digital cameras," in International Symposium on Multispectral Imaging and Color Reproduction for Digital Archives, pp. 42-49, 1999.

17. H.-L. Shen, P.-Q. Cai, S.-J. Shao, and J. H. Xin, "Reflectance reconstruction for multispectral imaging by adaptive wiener estimation," Opt. Express, vol. 15, pp. 15545-15554, Nov 2007. 